This copy is for your personal, non-commercial use only.

If you wish to distribute this article to others, you can order high-quality copies for your colleagues, clients, or customers by clicking here.

Permission to republish or repurpose articles or portions of articles can be obtained by following the guidelines here.

The following resources related to this article are available online at www.sciencemag.org (this information is current as of May 26, 2013 ):

Updated information and services, including high-resolution figures, can be found in the online version of this article at:

http://www.sciencemag.org/content/340/6135/987.full.html

Supporting Online Material can be found at:

http://www.sciencemag.org/content/suppl/2013/05/22/340.6135.987.DC1.html

This article cites 81 articles, 22 of which can be accessed free:

http://www.sciencemag.org/content/340/6135/987.full.html\#ref-list-1

This article appears in the following subject collections:

Biochemistry

http://www.sciencemag.org/cgi/collection/biochem

Medicine, Diseases

http://www.sciencemag.org/cgi/collection/medicine 
phosphate cytidylyltransferase (PF13_0253)also correlate with parasite survival in two other $P$. falciparum strains (Fig. 2B, black arrows, and table S4). The GB4 SNP alleles are shared with the NF54 and 3D7 strains that also survive, and the 7G8 SNP alleles are shared with the SL strain that is melanized by the $\mathrm{R}$ strain (7). Five genes were selected as top candidates for detailed genetic analysis on the basis of large differences in gene expression and/or on polymorphisms that correlates with survival in other strains (table S5).

Two top candidate genes, $P f_{S 4} 4$ and $P f_{S 48} /$ 45 , code for members of the 6-cysteine protein family that are expressed on the gametocyte surface. Previous gene disruption experiments in the NF54 line revealed that $P f_{s} 48 / 45$ is critical for gamete fertility (12). Pfs 47 is expressed in female gametocytes but is not essential for $P$. falciparum fertilization, although its homolog in Plasmodium berghei is required for female gamete fertility $(12,13)$. The intensity of infection with the $P f s 48 / 45$ knockout (KO) line (NF54 genetic background) in the $\mathrm{R}$ strain was low, probably because of reduced fertility. However, those parasites that invaded the midgut had a similar phenotype as wild-type (WT) NF54 parasites (8), and only $3 \%$ were melanized (fig. S4), indicating that $P f_{s} 48 / 45$ is not required to evade the mosquito immune system. In contrast, $P f_{S} 47 \mathrm{KO}$ (NF54 genetic background) parasites develop and invade the midgut but are eliminated by R mosquitoes ( $99 \%$ melanization) (Fig. 3A); although $P f_{s} 47 \mathrm{KO}$ parasites are not melanized by $\mathrm{S}$ mosquitoes (Fig. 3A), the infection level in the $\mathrm{S}$ strain (median of one oocyst per midgut) is much lower than that in $A$. stephensi mosquitoes (60 oocysts per midgut median) (fig. S5). This A. stephensi strain has been selected to be highly permissive to $P$. falciparum infection (14).

To determine whether $P f_{S} 47$ interacts with the mosquito immune system, we disrupted the A. gambiae complement-like system by silencing TEP1. Reducing TEP1 expression completely reversed melanization of $P f_{s} 47 \mathrm{KO}$ parasites in the $\mathrm{R}$ strain (Fig. 3B). In the A. gambiae $\mathrm{S}$ strain (G3), neither NF54 WT nor Pfs $47 \mathrm{KO}$ parasites were melanized (Fig. 3C); although TEP1 silencing had no significant effect on infection with NF54 WT parasites (Fig. 3C), it increased both the intensity $(P<0.0001$, Mann-Whitney test $)$ and the prevalence of infection $\left(P<0.001 ; \chi^{2}\right.$ test $)$ of $P f_{S} 47$ KO parasites (Fig. $3 \mathrm{C}$ ). This result indicates that $P f_{s} 47$ is necessary for $P$. falciparum parasites to evade two well-characterized immune responses mediated by TEP1 in A. gambiae: killing followed by melanization in the $\mathrm{R}$ strain and parasite lysis without melanization in the $\mathrm{S}$ strain.

$P f_{s} 47$ protein is present on the surface of WT NF54 ookinetes, the stage that invades the midgut, but is absent in $P f_{s} 47 \mathrm{KO}$ parasites (Fig. 3D). The expression of HPX2 and NOX5, two enzymes that mediate midgut nitration in response to $P$. berghei infection and promote TEP1 activation (2), was evaluated in S mosquitoes. HPX2 and NOX5 were not induced by NF54 WT parasites, and nitration levels were lower than in uninfected controls (Fig. 3E). In contrast, $P f_{s} 47 \mathrm{KO}$ parasites induced expression of HPX2 and NOX5 and a robust nitration response, indicating that $P f s 47$ may prevent TEP1-mediated lysis by suppressing midgut epithelial nitration responses (Fig. 3E).

Finally, we confirmed the importance of $P f_{s} 47$ for parasite survival by complementing the Pfs 47 $\mathrm{KO}$ line with different $P f s 47$ alleles (figs. S6 to S9). As expected, the NF54 allele of $P f s 47$ reversed the melanization phenotype ( $0 \%$ melanization) in the $\mathrm{R}$ strain when the complemented parasites were kept under sustained drug pressure, confirming that this allele of $P f_{s} 47$ is sufficient to evade the immune system (Fig. 4A). A reversal to a mixed live/melanization phenotype was observed when the drug pressure was reduced (fig. S10). In contrast, complementation with the 7G8 allele failed to rescue parasites in the R strain, because $99 \%$ melanization was observed (Fig. 4B).

Together, our findings identify $P f_{s} 47$ as an essential survival factor for $P$. falciparum that allows the parasite to evade the immune system of A. gambiae, a major mosquito vector in Africa. However, other parasite genes may also be involved in this process. Pfs 47 is a highly polymorphic gene with a marked population structure in field isolates and exhibits extreme fixation in non-African regions of the world $(15,16)$. Our findings suggest that the population structure of $P f s 47$ may be due to adaptation of $P$. falciparum to the different Anopheles vector species present outside of Africa. The fact that the 7G8 allele of $P f s 47$ is sufficient to evade the TEP1 complementlike system in $\mathrm{S}$ mosquitoes but not in the $\mathrm{R}$ strain indicates that there are also genetic differences in the vector that determine compatibility with parasites that express specific $P f_{s} 47$ alleles. It appears that $P f s 47$ evolved a function in $P$. falciparum that increases parasite survival in A. gambiae mosquitoes and may be responsible, at least in part, for the very high rates of malaria transmission in hyperendemic regions in Africa. Disruption of the immunomodulatory activity of Pfs 47 may prove to be an effective strategy to reduce malaria transmission to humans.

References and Notes

1. S. Kumar, L. Gupta, Y. S. Han, C. Barillas-Mury, J. Biol. Chem. 279, 53475 (2004).

2. G. A. Oliveira, J. Lieberman, C. Barillas-Mury, Science 335, 856 (2012)

3. S. Blandin et al., Cell 116, 661 (2004).

4. L. S. Garver, Y. Dong, G. Dimopoulos, PLoS Pathog. $\mathbf{5}$ e1000335 (2009).

5. C. Mitri et al., PLoS Pathog. 5, e1000576 (2009).

6. A. Cohuet et al., EMBO Rep. 7, 1285 (2006)

7. F. H. Collins et al., Science 234, 607 (1986).

8. A. Molina-Cruz et al., Proc. Natl. Acad. Sci. U.S.A. 109, E1957 (2012)

9. K. Hayton et al., Cell Host Microbe 4, 40 (2008).

10. Supplementary materials are available on Science Online.

11. R. Carter, P. Hunt, S. Cheesman, Int. J. Parasitol. 37, 285 (2007).

12. M. R. van Dijk et al., Cell 104, 153 (2001).

13. B. C. van Schaijk et al., Mol. Biochem. Parasitol. 149, 216 (2006)

14. A. M. Feldmann, T. Ponnudurai, Med. Vet. Entomol. 3, 41 (1989).

15. T. G. Anthony, S. D. Polley, A. P. Vogler, D. J. Conway, Mol. Biochem. Parasitol. 156, 117 (2007).

16. M. Manske et al., Nature 487, 375 (2012).

Acknowledgments: This work was supported by the Division of Intramural Research, National Institute of Allergy and Infectious Diseases, NIH. We thank K. Hayton and T. Wellems for advice with the genetic cross, A. Laughinghouse and K. Lee for technical support, and B. Marshall for editorial assistance. This work is subject to U.S. Patent no. 61/684,333, filed 17 August 2012, "Transmission-blocking malaria vaccine." There are material transfer agreements or patents restricting use of Pfs $48 / 45$ and Pfs 47 KO parasites.

\section{Supplementary Materials}

wWw.sciencemag.org/cgi/content/ful//science.1235264/DC1 Materials and Methods

Figs. $\$ 1$ to 59

Tables $\mathrm{S} 1$ to $\mathrm{S6}$

References (17-28)

16 January 2013; accepted 11 April 2013

Published online 9 May 2013;

10.1126/science.1235264

\title{
Tetrahydrobiopterin Biosynthesis as an Off-Target of Sulfa Drugs
}

\author{
Hirohito Haruki, ${ }^{1}$ Miriam Grønlund Pedersen, ${ }^{1}$ Katarzyna Irena Gorska, ${ }^{1}$ \\ Florence Pojer, ${ }^{2}$ Kai Johnsson ${ }^{1 *}$
}

The introduction of sulfa drugs for the chemotherapy of bacterial infections in 1935 revolutionized medicine. Although their mechanism of action is understood, the molecular bases for most of their side effects remain obscure. Here, we report that sulfamethoxazole and other sulfa drugs interfere with tetrahydrobiopterin biosynthesis through inhibition of sepiapterin reductase. Crystal structures of sepiapterin reductase with bound sulfa drugs reveal how structurally diverse sulfa drugs achieve specific inhibition of the enzyme. The effect of sulfa drugs on tetrahydrobiopterin-dependent neurotransmitter biosynthesis in cell-based assays provides a rationale for some of their central nervous system-related side effects, particularly in high-dose sulfamethoxazole therapy of Pneumocystis pneumonia. Our findings reveal an unexpected aspect of the pharmacology of sulfa drugs and might translate into their improved medical use.

etrahydrobiopterin $\left(\mathrm{BH}_{4}\right)$ is a cofactor of aromatic hydroxylases, such as phenylalanine, tyrosine and tryptophan hydrox- ylases, all isoforms of nitric oxide synthases, and alkylglycerol monooxygenase (1). $\mathrm{BH}_{4}$ deficiencies result in symptoms of hyperphenyl- 
alaninemia, as well as dopamine and serotonin (5-hydroxytryptamine) deficiencies $(2,3)$. The final step in $\mathrm{BH}_{4}$ biosynthesis is the reduction of 6-pyruvoyltetrahydropterin by sepiapterin reductase (SPR) (Fig. 1A). $\mathrm{BH}_{4}$ is also recycled from the oxidized form of the cofactor and synthesized by a salvage pathway via dihydrobiopterin $\left(\mathrm{BH}_{2}\right)($ Fig. 1A). Recently, we showed that the anti-inflammatory drug sulfasalazine and its metabolite sulfapyridine are potent inhibitors of SPR and proposed that an inhibition of $\mathrm{BH}_{4}$ biosynthesis by the highly permeable sulfapyridine is responsible for the anti-inflammatory activity of sulfasalazine in rheumatoid arthritis (4). Here, we report that the inhibition of $\mathrm{BH}_{4}$ biosynthesis contributes to the pharmacological properties of other clinically approved drugs. By screening a library of approved drugs for SPR inhibitors, we identified 10 drugs that inhibited SPR with median inhibitory concentration $\left(\mathrm{IC}_{50}\right)$ values below $100 \mathrm{nM}$ (Fig. 1B and tables S1 and S2). These included the known SPR inhibitors sulfasalazine and sulfapyridine. Furthermore, the antibacterial sulfa drugs - sulfathiazole, sulfamethoxazole, sulfamethizole, phtalylsulfathiazole, and sulfadiazine, as well as the three antidiabetic sulfonylureas chlorpropamide, glibenclamide, and tolbutamidewere identified as potent SPR inhibitors. To gain an understanding of the binding mode of the different sulfonamides to SPR, we solved the crystal structures of SPR with bound nicotinamide adenine dinucleotide phosphate $\left(\mathrm{NADP}^{+}\right)$and sulfapyridine or sulfathiazole. SPR, NADP ${ }^{+}$, and sulfapyridine or sulfathiazole form a ternary complex in which the sulfa drugs block the substrate binding site (Fig. 2, A and B). The two drugs bind to SPR in a near-identical fashion (Fig. 2B), and there are no substantial differences detectable between the two protein structures (root-mean-square deviation between backbone $\mathrm{C} \alpha$ atoms of $0.1 \AA$ ). The sulfonamide, the moiety common to all hits, and the nitrogen atom in the heteroaromatic ring of sulfapyridine and sulfathiazole form specific hydrogen bonds with active site residues $\mathrm{Ser}^{157}, \mathrm{Tyr}^{170}$, and $\mathrm{Asp}^{257}$ (Fig. 2C). A hydrogen bond acceptor at a position equivalent to that of the nitrogen atom in the pyridine ring of sulfapyridine is found in all identified SPR inhibitors (Fig. 1B); in the sulfonylureas, the oxygen atom of the urea group could function as a hydrogen bond acceptor. Previous structural studies on mouse SPR have revealed that these three residues are important for substrate binding (5). The pyridine or thiazole rings also stack on top of the nicotinamide ring of $\mathrm{NADP}^{+}$(Fig. 2B) and make hydrophobic contacts with residues Leu ${ }^{104}, \operatorname{Trp}^{167}, \mathrm{Met}^{205}$, and $\mathrm{Ala}^{209}$. The different heterocycles or alkyl groups

${ }^{1}$ École Polytechnique Fédérale de Lausanne (EPFL), Institute of Chemical Sciences and Engineering, Institute of Bioengineering, National Centre of Competence in Research (NCCR) in Chemical Biology, 1015 Lausanne, Switzerland. ²EPFL, Global Health Institute, 1015 Lausanne, Switzerland.

*Corresponding author. E-mail: kai.johnsson@epfl.ch found in other SPR inhibitors (Fig. 1B) should be able to bind in an equivalent fashion as the heteroaromatic ring of sulfapyridine and sulfathiazole. Our structural data thus suggest how structurally diverse sulfonamides achieve inhi- bition of SPR but also why certain sulfa drugs, such as sulfamethazine (Fig. 1B), are only very weak inhibitors of SPR. Sulfamethazine is a weaker inhibitor of SPR than sulfadiazine, which is 370 times as strong, although it differs from

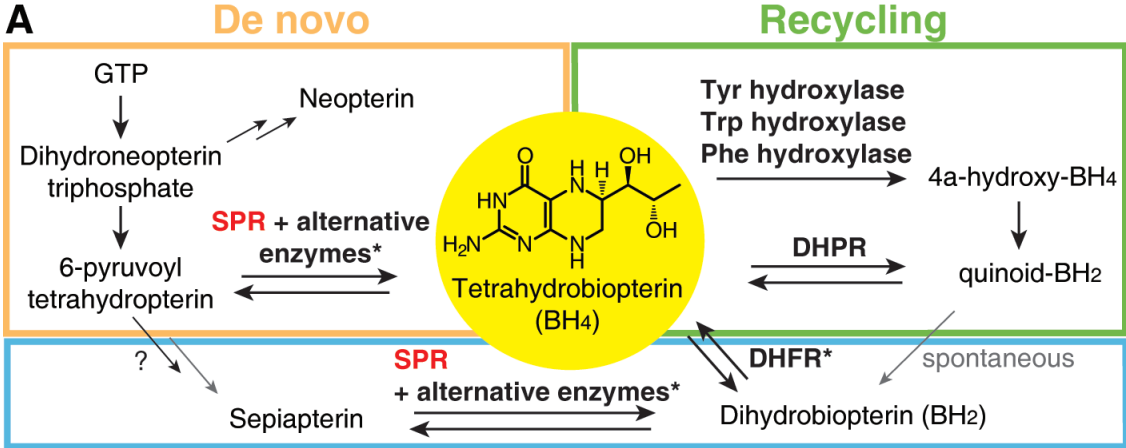

B

Salvage

* Low activity in brain

\begin{tabular}{|c|c|c|c|c|}
\hline Drug & Chemical structure & $\begin{array}{c}\mathrm{IC}_{50} \text { in enz. } \\
\text { assay } \\
\text { [nM] }\end{array}$ & $\begin{array}{c}\text { Average } C_{\mathrm{ss}} \\
\text { at therapeutic } \\
\text { dose } \dagger[\mu \mathrm{M}]\end{array}$ & $\begin{array}{c}\text { Ratio of } \\
\text { CSF-to-blood } \\
\text { conc. } \dagger\end{array}$ \\
\hline Sulfasalazine & & 7 & $<50$ & n.a. \\
\hline Sulfathiazole & & 14 & $200-400$ & $0.1-0.2$ \\
\hline Sulfamethoxazole & & 19 & $\begin{array}{c}600-1,300 \\
\text { (for PCP) }\end{array}$ & $0.2-0.5$ \\
\hline Sulfamethizole & & 19 & $200-400$ & n.a. \\
\hline Phthalylsulfathiaz & & 24 & $<50$ & n.a. \\
\hline Chlorpropamide & & 36 & mean 260 & n.a. \\
\hline Sulfadiazine & & 43 & $200-400$ & 0.3 \\
\hline Sulfapyridine & & 82 & $\begin{array}{c}100-200 \\
\text { (at } 2-4 \text { g/day } \\
\text { sulfasalazine) }\end{array}$ & $0.6-0.7$ \\
\hline Glibenclamide & & 82 & $<1$ & n.a. \\
\hline Tolbutamide & & 92 & mean 100 & $<0.1$ \\
\hline $\begin{array}{l}\text { Sulfamethazine } \\
\text { (negative control) }\end{array}$ & & $16^{\prime} 000$ & $200-400$ & $0.4-0.6$ \\
\hline
\end{tabular}

$\dagger$ Literature values

Fig. 1. Pathways for $\mathbf{B H}_{4}$ homeostasis and structures of SPR inhibitors. (A) $\mathrm{BH}_{4}$ biosynthetic pathways. SPR catalyzes formation of $\mathrm{BH}_{4}$ from 6-pyruvoyltetrahydropterin and of $\mathrm{BH}_{2}$ from sepiapterin in a salvage pathway. (B) SPR inhibitors, their $\mathrm{IC}_{50}$ values for inhibition of SPR in an enzymatic assay, and some of their pharmacokinetic properties. $I C_{50}$ values are means of triplicate experiments $(N=2$ at least) (full data in table S2). The benzenesulfonamide moiety found in all SPR inhibitors is highlighted in red. Average steady-state serum or blood concentrations $\left(C_{\mathrm{ss}}\right)$ at therapeutic dose and the ratio of CSF-toblood concentrations are literature values (see references in table S3). The listed ratio of CSF-to-blood concentration of tolbutamide was determined in rats $(26,27)$. Sulfamethazine, which only weakly inhibits SPR, served as negative control throughout this work. n.a., not available. 
sulfadiazine only by two additional methyl groups attached to the pyrimidine ring (Fig. 1B). A manual superimposition of sulfamethazine on sulfapyridine in the structure of SPR reveals an energetically unfavorable interaction of the two additional methyl groups with the side chains of $\mathrm{Met}^{205}$ and Gln ${ }^{206}$, respectively; the distance of the sulfur atom of $\mathrm{Met}^{205}$ and the amide ni-
A

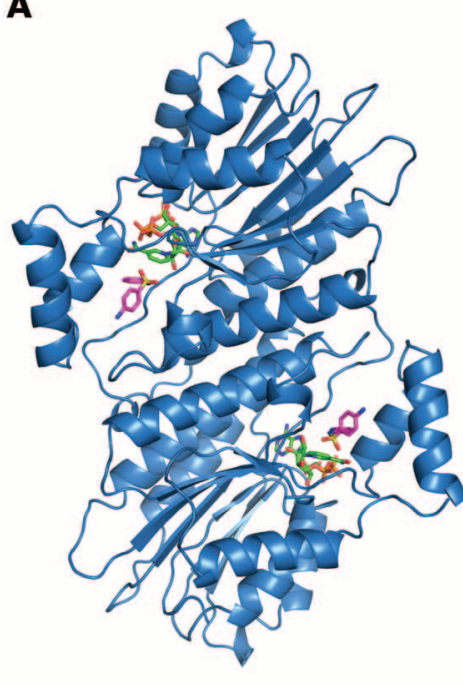

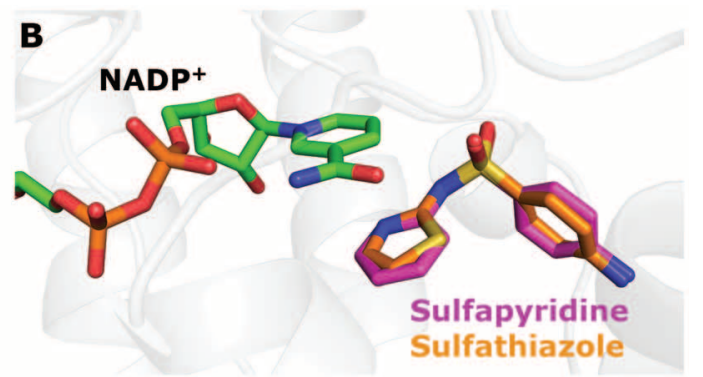

C

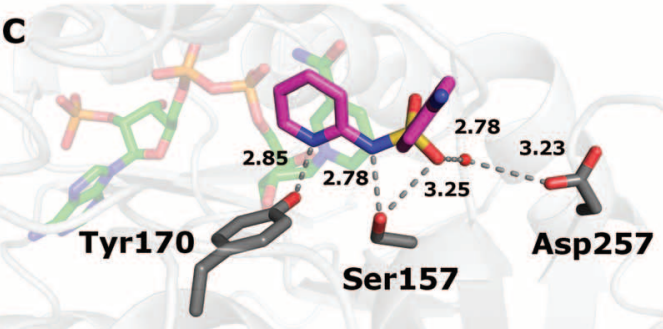

Fig. 2. Binding mode of sulfa drugs to SPR. (A) Overview of crystal structure of human SPR in complex with $\mathrm{NADP}^{+}$(green) and sulfapyridine (magenta). (B) Close-up of a $2.4 \AA$ crystal structure of human SPR with bound $\mathrm{NADP}^{+}$and sulfapyridine. In the ternary complex, the pyridine ring of sulfapyridine is stacked on top of the nicotinamide ring of NADP ${ }^{+}$with a distance of $3.3 \AA$ between the nitrogen in the pyridine ring and $\mathrm{C} 4$ of the nicotinamide ring. The overlay of sulfapyridine with sulfathiazole (orange) from a $2.45 \AA$ crystal structure of human SPR with bound NADP ${ }^{+}$and sulfathiazole shows that the two drugs bind to SPR in a near-identical fashion. (C) Key hydrogen bonds between the sulfonamide moiety of sulfapyridine and active site residues $\operatorname{Ser}^{157}, \operatorname{Tyr}^{170}$, and $\operatorname{Asp}^{257}$ known to be important for substrate binding (5). A water molecule (red) mediates the hydrogen bond between $\mathrm{Asp}^{257}$ and sulfapyridine. Distances are given in $\AA$.

trogen of $\mathrm{Gln}^{206}$ to the nearest methyl group is $2.55 \AA$ and $2.75 \AA$, respectively (fig. S1, D and E). These distances are more than $0.5 \AA$ below the corresponding nonbonded contact distances observed in organic crystals and protein structures $(6,7)$.

Next, we examined if these drugs reduce $\mathrm{BH}_{4}$ levels in human primary dermal fibroblasts in which $\mathrm{BH}_{4}$ biosynthesis is induced by proinflammatory cytokines. Incubation of human fibroblasts with interferon- $\gamma($ IFN- $\gamma)$ and tumor necrosis factor- $\alpha$ (TNF $\alpha)$ for 24 hours increased $\mathrm{BH}_{4}$ levels 165 -fold to $0.33 \mathrm{nmol} / \mathrm{mg}$ protein (Fig. 3A). SPR inhibitors decreased $\mathrm{BH}_{4}$ levels in cytokine-stimulated fibroblasts in a dosedependent manner, whereas sulfamethazine had no effect on $\mathrm{BH}_{4}$ levels. Dihydroneopterin triphosphate (DHNTP) is a precursor of the SPR substrate 6-pyruvoyltetrahydropterin in the biosynthesis of $\mathrm{BH}_{4}$ (Fig. 1A), and increased DHNTP levels have been detected in cytokine-stimulated fibroblasts derived from SPR-deficient patients (8). Accordingly, treatment with SPR inhibitors significantly increased DHNTP levels (measured as neopterin after phosphatase treatment) (Fig. 3A and Fig. 1A), which confirmed that the observed decrease in $\mathrm{BH}_{4}$ is due to an inhibition of SPR. Note that, the observed inhibition of $\mathrm{BH}_{4}$ biosynthesis occurs at drug concentrations that are within their reported serum levels (Fig. 1B). The exception in these experiments was sulfasalazine, which decreased $\mathrm{BH}_{4}$ levels only at high micromolar concentrations and mainly by inhibition of cytokine signaling (fig. S2). The low potency of sulfasalazine as SPR inhibitor in cell-based assays can be attributed to its low cell permeability (9).

Fig. 3. Inhibition of $\mathrm{BH}_{4}$ and L-dopa synthesis by sulfonamides. (A) Effect of selected drugs on $\mathrm{BH}_{4}$ synthesis in cytokine-stimulated human primary dermal fibroblasts. Intracellular $\mathrm{BH}_{4}$ and neopterin levels were measured after 24 hours concomitant treatment of IFN- $\gamma$ and TNF $\alpha$ and SPR inhibitors $(0.01,0.1$, and $1 \mathrm{mM})$. Data are means \pm SD from triplicate experiments $[N=1$, except $N=$ 3 for dimethyl sulfoxide (DMSO) + cytokines]. (B) Effect of selected drugs on $\mathrm{BH}_{4}$ and extracellular 3-MT levels in SK-N-BE(2) cells. Cells were incubated

$A$
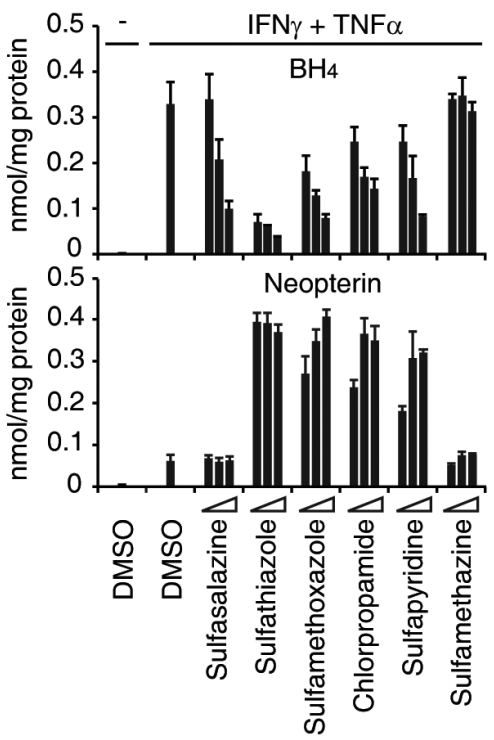

B

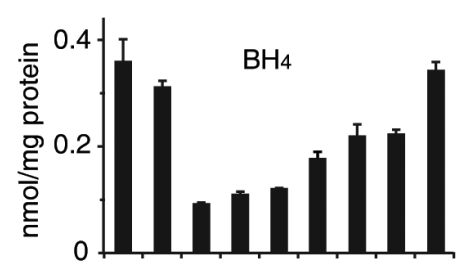

4 - $3-\mathrm{MT}$

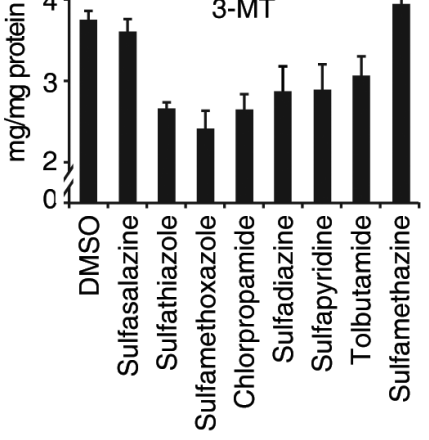

C

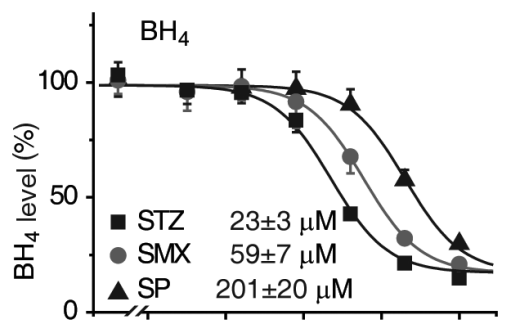

with drugs for 24 hours at $200 \mu \mathrm{M}$ concentrations. Intracellular $\mathrm{BH}_{4}$ level and 3-MT level in culture supernatant were measured. Data are means \pm SD from triplicate experiments $(N=1)$. (C) Effect of sulfamethoxazole (SMX), sulfathiazole (STZ), and sulfapyridine (SP) on $\mathrm{BH}_{4}$ synthesis and L-dopa levels in SK-N-BE(2) cells. Intracellular $\mathrm{BH}_{4}$ levels were measured after 24-hour treatment of cells with drugs. Extracellular L-dopa levels were measured after 24-hour treatment of cells with drugs, followed by $30 \mathrm{~min}$ of treatment with $10 \mu \mathrm{M}$ NSD-1015, aromatic L-amino acid decarboxylase inhibitor. Data are means \pm SD from triplicate experiments $(N=1,2,3$ for STZ, SP, and SMX, respectively). 
Sulfamethoxazole is currently the most-used sulfa drug and is prescribed in combination with trimethoprim (cotrimoxazole) for microbial infections and as standard treatment against Pneumocystis pneumonia (PCP), a prevalent opportunistic fungal infection in immunocompromised individuals $(10,11)$. Both sulfamethoxazole and trimethoprim target pathogen tetrahydrofolate synthesis; sulfamethoxazole is a competitive inhibitor of dihydropteroate synthase, whereas trimethoprim inhibits dihydrofolate reductase (DHFR). Adverse effects of sulfamethoxazoletrimethoprim at standard dose for bacterial infection are relatively rare, but CNS-related side effects are frequently observed at the higher therapeutic dose used in PCP (up to $100 \mathrm{mg} / \mathrm{kg}$ of body weight per day sulfamethoxazole and $20 \mathrm{mg} / \mathrm{kg}$ per day of trimethoprim). These include anorexia, nausea, headache, nervousness, fine tremors, lightheadedness, insomnia, and drowsiness (see table $\mathrm{S} 4$ for a compilation of reports concerning CNS-related adverse effects). For example, administering $100 \mathrm{mg} / \mathrm{kg}$ per day of sulfamethoxazole to 12 healthy adults resulted in CNS-related adverse effects in all subjects within 3 days (12). Furthermore, recent analyses of clinical data of PCP patients indicate that highdose sulfamethoxazole-trimethoprim therapy can cause psychosis; the reported incidence being 12 to $20 \%(13,14)$. Nausea, vomiting, and CNSrelated symptoms are common, dose-dependent adverse effects of most sulfa drugs (15-17). In sulfasalazine treatment, these symptoms are correlated with serum concentrations of sulfapyridine but not sulfasalazine (18). In dogs, which exhibit CNS-related side effects similar to those of humans (19), complete gastrointestinal evisceration or direct application of sulfapyridine to the vomiting center showed that nausea and vomiting are not caused by direct action of the drug on the gastrointestinal tract or the vomiting center (20). The inhibition of $\mathrm{BH}_{4}$ biosynthesis by sulfa drugs and a subsequent inhibition of neurotransmitter biosynthesis could provide a rationale for their CNS-related side effects. This hypothesis is supported by the observation that the adverse effects of sulfamethoxazole resemble the symptoms of genetically inherited SPR deficiency. Basal levels of $\mathrm{BH}_{4}$ in SPR deficiency in most tissues are maintained through salvage or alternative pathways (Fig. 1A). An exception is the brain, where the low expression level of the enzymes involved in salvage pathways, such as DHFR, diminishes their efficiency. Symptoms of SPR deficiency are those of catecholamine and serotonin deficiencies with disabling motor and cognitive deficits (2). Furthermore, recurrent vomiting is a gastrointestinal symptom of SPR deficiency (2). SPR deficiency can be most reliably diagnosed through analysis of cerebrospinal fluid (CSF); CSF concentrations of $\mathrm{BH}_{2}$ and sepiapterin in SPR deficiency are significantly increased whereas those of dopamine and serotonin metabolites are decreased (2). An effect of sulfamethoxazole-trimethoprim on biopterin levels in CSF has been reported previously: Patients with Machado-Joseph disease, an autosomal dominantly inherited neurodegenerative disorder, who were administered sulfamethoxazoletrimethoprim (400 mg sulfamethoxazole, $80 \mathrm{mg}$ trimethoprim, twice daily) showed significantly increased $\mathrm{BH}_{2}$ levels in CSF (21). Dopamine insufficiency has been reported in a child with dihydropteridine reductase (DHPR) deficiency after being given sulfamethoxazole-trimethoprim (22). DHPR is involved in recycling of $\mathrm{BH}_{4}$ (Fig. 1A), and the observed dopamine insufficiency could be explained by a further depletion of already decreased $\mathrm{BH}_{4}$ levels through inhibition of SPR.

To demonstrate that sulfa drugs can lower neurotransmitter levels through depletion of $\mathrm{BH}_{4}$, we investigated whether sulfa drugs in cell culture experiments would affect catecholamine biosynthesis in the human neuroblastoma cell line SK-N-BE(2) (23). SK-N-BE(2) cells have constitutively high levels of $\mathrm{BH}_{4}$ and excrete substantial amounts of the dopamine metabolite 3-methoxytyramine (3-MT). The identified SPR inhibitors, with the exception of sulfasalazine, at $200 \mu \mathrm{M}$, decreased intracellular $\mathrm{BH}_{4}$ and extracellular 3-MT levels (Fig. 3B). The activities in this cellular assay, again with the exception of sulfasalazine, correlated well with the $\mathrm{IC}_{50}$ values measured in the enzymatic assay (Figs. 1B and $3 \mathrm{~B})$. For more detailed analysis, SK-N-BE(2) cells were incubated with varying concentrations of sulfathiazole, sulfamethoxazole, or sulfapyridine; the drugs decreased $\mathrm{BH}_{4}$ levels from 0.3 to $0.05 \mathrm{nmol} / \mathrm{mg}$ protein, with $\mathrm{IC}_{50}$ values of 23, 59, and $201 \mu \mathrm{M}$, respectively (Fig. 3C). To examine tyrosine hydroxylase activity in drug-treated cells, extracellular L-dopa levels were measured after 24 hours of treatment with sulfathiazole, sulfamethoxazole, or sulfapyridine (Fig. 3C) (24). In these experiments, sulfamethoxazole, sulfathiazole, and sulfapyridine decreased L-dopa levels with $\mathrm{IC}_{50}$ values of 31 , 63 , and $143 \mu \mathrm{M}$, respectively. In conclusion, sulfa drugs at concentrations below their serum levels interfere with catecholamine biosynthesis through $\mathrm{BH}_{4}$ depletion. We assume that similar effects will be observed on serotonin biosynthesis.

Our findings, together with ample clinical observations, suggest that inhibition of $\mathrm{BH}_{4}$ biosynthesis by sulfa drugs is responsible for some of their CNS-related side effects. The incidence of these side effects will depend on the inhibitory activity of the sulfa drug toward SPR - its serum concentration during therapy, as well as its uptake into the CNS. Sulfamethoxazole reaches plasma levels of up to $600 \mu \mathrm{M}$, shows good uptake into the CNS, and is a potent inhibitor of SPR in cellular assays (Fig. 1B and Fig. 3); we therefore conclude that sulfamethoxazole at these doses will affect $\mathrm{BH}_{4}$ and catecholamine biosynthesis in the brain. The excellent CNS uptake of sulfapyridine and its good inhibitory activity against SPR explain why its CNS-related side effects are already observed at lower serum concentrations compared with other sulfa drugs also inhibiting
SPR (i.e., sulfadiazine). Furthermore, sulfa drugs not inhibiting SPR also show diminished CNSrelated side effects. In early studies on the efficacy of sulfamethazine in treatment of labor pneumonia (73 patients), it was noted that "The striking difference, however, was that the patients never complained of the severe mental and physical depression which often accompanies sulfapyridine treatment" (25). CNS-related side effects have not been published for first-generation sulfonylureas tolbutamide and chlorpropamide and second-generation sulfonylurea glibenclamide. The effective serum concentration of glibenclamide is too low for SPR inhibition (Fig. 1B). However, the serum concentrations of tolbutamide and chlorpropamide are comparable to those measured for sulfa drugs. We assume that low CNS uptake of these drugs is responsible for the absence of such side effects. In fact, tolbutamide uptake in rat CNS is extremely low compared with other drugs $(26,27)$.

Sulfa drugs were the scaffold for the development of multiple other drugs (28). Proteins that are targeted by such drugs - for example, carbonic anhydrase and endothelin receptor-are therefore possible off-targets of sulfa drugs, and some carbonic anhydrase inhibitors (sultiame) or endothelin receptor antagonists (sitaxentan) cause side effects such as headache and dizziness. We could not observe inhibition of human carbonic anhydrase II, a representative isozyme of carbonic anhydrases, by sulfa drugs $\left(\mathrm{IC}_{50}>\right.$ $1 \mathrm{mM}$ in an enzymatic assay) (table S6). Sulfamethoxazole and sulfathiazole were reported to compete with endothelin-1 for binding to endothelin receptor type A with $\mathrm{IC}_{50}$ values that are within their therapeutic serum concentrations, but in functional assays sulfamethoxazole did not show activity at the highest concentrations tested $(100 \mu \mathrm{M})$ (table S7). These observations allow us to rule out carbonic anhydrase II but not endothelin receptor as potential additional off-targets. More generally speaking, our results do not exclude the presence of other off-targets of sulfa drugs.

Our finding that sulfa drugs interfere with $\mathrm{BH}_{4}$-dependent neurotransmitter biosynthesis also suggests strategies for a potential improvement of PCP therapy. L-Dopa or 5-hydroxytryptophan, which are successfully used as supplements in SPR deficiency (2), might dampen some of their CNS-related side effects in high-dose sulfamethoxazole therapy. Second, sulfa drugs displaying no, or weak, SPR inhibition should be considered as alternatives to sulfamethoxazole (29). In summary, the identification of sulfa drugs as inhibitors of $\mathrm{BH}_{4}$ biosynthesis is of relevance for understanding the history of sulfa drugs and has direct implications for their future use.

References and Notes

1. E. R. Werner, N. Blau, B. Thöny, Biochem. J. 438, 397 (2011)

2. ]. Friedman et al., Ann. Neurol. 71, 520 (2012).

3. N. Blau, ]. B. Hennermann, U. Langenbeck,

U. Lichter-Konecki, Mol. Genet. Metab. 104 (suppl.) S2 (2011).

4. C. Chidley, H. Haruki, M. G. Pedersen, E. Muller, K. Johnsson, Nat. Chem. Biol. 7, 375 (2011). 
5. G. Auerbach et al., EMBO J. 16, 7219 (1997).

6. D. Seeliger, B. L. de Groot, Proteins 68, 595 (2007).

7. R. S. Rowland, R. Taylor, J. Phys. Chem. 100, 7384 (1996).

8. L. Bonafé, B. Thöny, ]. M. Penzien, B. Czarnecki, N. Blau, Am. J. Hum. Genet. 69, 269 (2001)

9. T. Kenakin, A Pharmacology Primer (Elsevier, London, ed. 3, 2009), pp. 184-188.

10. ]. Helweg-Larsen, T. Benfield, C. Atzori, R. F. Miller J. Antimicrob. Chemother. 64, 1282 (2009).

11. M. Harigai, R. Koike, N. Miyasaka, N. Engl. J. Med. 357 1874 (2007)

12. R. C. Stevens, S. C. Laizure, C. L. Williams, D. S. Stein, Antimicrob. Agents Chemother. 35, 1884 (1991)

13. L. E. Walker et al., J. Antimicrob. Chemother. 66 1117 (2011)

14. K.-Y. Lee et al., J. Antimicrob. Chemother. 67, 2749 (2012).

15. A. E. Cribb, B. L. Lee, L. A. Trepanier, S. P. Spielberg, Adverse Drug React. Toxicol. Rev. 15, 9 (1996).
16. C. S. Keefer, N. Engl. J. Med. 226, 266 (1942).

17. H. B. van Dyke, Ann. N. Y. Acad. Sci. 44, 477 (1943).

18. T. Pullar, Adverse Drug React. Toxicol. Rev. 11, 93 (1992).

19. E. K. Marshall, ]. T. Litchfield, J. Pharmacol. Exp. Ther. 67, 454 (1939).

20. J. F. Sadusk, J. W. Hirshfeld, A. Seymour, Yale J. Biol. Med. 13, 351 (1941).

21. T. Sakai, T. Matsuishi, S. Yamada, H. Komori, H. Iwashita, J. Neural Transm. Gen. Sect. 102, 159 (1995).

22. R. C. Woody, M. A. Brewster, Dev. Med. Child Neurol. 32, 639 (1990)

23. J. L. Biedler, S. Roffler-Tarlov, M. Schachner, L. S. Freedman, Cancer Res. 38, 3751 (1978).

24. M. Bräutigam, R. Dreesen, H. Herken, J. Neurochem 42, 390 (1984).

25. D. W. Macartney, R. W. Luxton, G. S. Smith, W. A. Ramsay, J. Goldman, Lancet 239, 639 (1942).

26. O. Sugita, Y. Sawada, Y. Sugiyama, T. Iga, M. Hanano, ]. Pharmacokinet. Biopharm. 10, 297 (1982).

27. O. Lacombe et al., Mol. Pharm. 8, 651 (2011).
28. J. Drews, Science 287, 1960 (2000)

29. Y. L. Hong et al., J. Eukaryot. Microbiol. 43, 40 S (1996).

Acknowledgments: The authors thank G. Turcatti, M. Chambon, M. Busquets, and M. Rengifo González for technical assistance; the Swiss Light Source for support during x-ray data collection; and G. Lukinavicius, O. Sallin, P. Hauser, and C. Woolf for helpful discussions. This work was supported by EPFL, the Swiss National Science Foundation, and the NCCR Chemical Biology. Coordinates and structure factors for the crystal structures have been deposited in the Protein Data Bank (PDB), and the PDB accession codes are listed in table S8.

\section{Supplementary Materials}

www.sciencemag.org/cgi/content/full/340/6135/987/DC1

Materials and Methods

Figs. $\mathrm{S} 1$ to $\mathrm{S} 3$

Tables $\mathrm{S} 1$ to $\mathrm{S} 8$

References (30-82)

19 November 2012; accepted 8 April 2013

10.1126/science.1232972

\section{Defining Single Molecular Forces Required to Activate Integrin and Notch Signaling}

\author{
Xuefeng Wang ${ }^{1}$ and Taekjip $\mathrm{Ha}^{1,2 *}$
}

Cell-cell and cell-matrix mechanical interactions through membrane receptors direct a wide range of cellular functions and orchestrate the development of multicellular organisms. To define the single molecular forces required to activate signaling through a ligand-receptor bond, we developed the tension gauge tether (TGT) approach in which the ligand is immobilized to a surface through a rupturable tether before receptor engagement. TGT serves as an autonomous gauge to restrict the receptor-ligand tension. Using a range of tethers with tunable tension tolerances, we show that cells apply a universal peak tension of about 40 piconewtons $(\mathrm{pN})$ to single integrin-ligand bonds during initial adhesion. We find that less than $12 \mathrm{pN}$ is required to activate Notch receptors. TGT can also provide a defined molecular mechanical cue to regulate cellular functions.

$\mathrm{C}$ ells sense and respond to the mechanical properties of the surrounding extracellular matrix (ECM) and neighboring cells. Reciprocally, cells also apply force on the ECM and transmit mechanical signals to neighboring cells. These mechanical interactions activate intracellular signaling pathways and regulate such diverse processes as cell adhesion, polarization, migration, proliferation, and differentiation $(1,2)$. As a result, by tuning bulk mechanical properties like stiffness, texture, and geometry of the substrate, researchers have gained insight into processes such as stem cell differentiation (3) and tumor metastasis (4). Single-molecule force spectroscopy has been used to study various mechanosensitive membrane receptors, including integrin, cadherin, and Notch (5-8). However, these approaches cannot reveal the single-molecule forces required for physiological functions because they either measure collective forces exerted through

${ }^{1}$ Department of Physics, Center for the Physics of Living Cells and Institute for Genomic Biology University of Illinois at Urbana-Champaign, Urbana, IL 61801, USA. ${ }^{2}$ Howard Hughes Medical Institute, Urbana, IL 61801, USA.

*Corresponding author. E-mail: tjha@illinois.edu many molecules or probe molecular unbinding or unfolding forces only. More recently, fluorescence resonance energy transfer-based force sensors were developed and inserted to target sites to monitor cellular forces $(9,10)$, but great efforts must be taken to prepare the sensors and to track and interpret the fluorescence signal. Here, we describe a platform termed tension gauge tether (TGT) that allows us to determine the single-molecule forces required for mechanical signaling in cells.

In TGT, a ligand is covalently conjugated to a tether that ruptures at a critical force, which we term "tension tolerance" or $T_{\text {tol }}$, and is immobilized on a solid surface through the tether (Fig. 1). Cells are plated on the surface, and membrane receptors engage with and apply tension to the ligands. If signal activation through the receptor requires a molecular tension larger than $T_{\text {tol }}$, the tether will rupture, abolishing signal activation. In contrast, if the required tension is smaller than $T_{\text {tol }}$, the tether will endure, activating the receptor-mediated signaling. By engineering a series of tethers with different $T_{\text {tol }}$ values, the tension required for signal activation can be determined by observing receptor-regulated cell activities, which are usually much easier to detect than single-molecule events. Because each ligand is equipped with an individual tension gauge, the measurement is independent of the receptor or ligand density.

DNA is a good candidate for use as rupturable tether in TGT because force application geometry (fig. S1) strongly affects its rupture force, with an unzipping force rupturing the DNA at a much lower force than a shearing force $(11,12)$. Albrecht et al. have exploited this feature to estimate the rupture force of an antigen-antibody bond relative to the rupture forces of double-stranded DNA (dsDNA) in unzipping or shear geometry (13). The estimated rupture force of a 21-base pair (bp) DNA is about $12 \mathrm{pN}$ in the unzipping geometry and is about $56 \mathrm{pN}$ in the shear geometry (14). Intermediate rupture forces can be obtained by applying forces through an internal position on the DNA duplex, and the theoretical $T_{\text {tol }}$ values of the resulting tethers can be estimated (Fig. 1D and fig. S2). $T_{\text {tol }}$ represents the force required to rupture the tether in less than $2 \mathrm{~s}$ when the force is applied at a constant level (12) [see supplementary text (14)].

Integrins are membrane receptors that mediate cell adhesion and sense and transduce mechanical information from the ECM into cells. Bulk traction forces applied through a collection of integrin-ligand bonds have been extensively examined (15-18). Here, we apply the TGT platform to probe the tension on a single integrin-ECM ligand bond required for cell adhesion (specifically, integrin $\alpha_{v} \beta_{3}$ and its ligand, cyclic RGDfK peptide). To reduce nonspecific adhesion, ligands with the DNA tether are immobilized through an Avidin-biotin linker on a glass surface passivated with polyethylene glycol (PEG). The Avidinbiotin unbinding force, $\sim 160 \mathrm{pN}$ (19), is much larger than the tether rupture forces used here $(\leq 56 \mathrm{pN})$.

We conjugated RGDfK to nine different DNA tethers with estimated $T_{\text {tol }}$ values of 12, 16, 23, $33,43,50,54,55$, and $56 \mathrm{pN}$ (fig. S2). These DNA tethers have various force application geometries but share the same length, sequence, and thermal stability. ATGT array was created by 